\title{
Dementia in Nepal: Early Recognition Knowledge, Management Barriers and Practices of Registered Nurses - A Pilot Study
}

\section{Pathak KP ${ }^{1}$ and Gaire $\mathrm{T}^{2 *}$}

${ }^{1 *}$ Faculty of Social Sciences, Nepal Open University, Lalitpur, Nepal; ${ }^{2}$ Department of Nursing, Innovative College Of Health Science, Kathmandu Nepal

\begin{abstract}
Background: Nursing staffs working in private nursing homes may not have the same degree of access to support and education on the dementia early recognition knowledge, management barriers and practices. Early recognition and undetected dementia in nursing home is a global problem. Since nurses can act as the first step in the identification process, management and care and help us to enhance the currently low recognition rates.

Aim: The aim of this research was to examine the early recognition knowledge, barriers and practices concerning registered nurses' management and care.

Methods: A purposive sampling approach with 44 registered nurses using structured multiple-choice questionnaire covering demographic questions, knowledge, management and caring barriers in the newly established nursing homes.

Results: Knowledge of Nurses with regard to the diagnosis, management and care of dementia was unsatisfactory $($ mean $=3.54, \mathrm{SD}=1.82)$. The care and management of patients with dementia in nursing homes settings is a challenge in current health care delivery.

Conclusion: Educational programs and health care policies that can help increase awareness of dementia in nursing practice, management and care to improve the care provided to dementia-afflicted people of Nepal.
\end{abstract}

Keywords: Dementia; Care givers (carers); Elderly; Registered Nurses (RNs)

\section{INTRODUCTION}

Dementia is a burden issue- not only for family member, but also for the health care professional due to its worldwide, dramatically increasing prevalence rate [1]. The estimation of worldwide prevalence of dementia is 24 million and its ratio may reach 40 million by 2020 , and 80 million by 2040 . Mainly the prevalence of dementia seems higher in developed countries than in lowand middle-income countries [2]. However, Marrin, et al., reveals that in $2010,58 \%$ of people with dementia will remain in low- or middle-income countries and this proportion will rise respectively to $63 \%$ in 2030 and $71 \%$ in 2050 [3].

If we only talk about dementia, it is a challenging issue of management and care for health professional due to its overlapping signs and symptoms, and chronic mental health condition. In the US, 5.2 million people have dementia-related disorders (especially Alzheimer dementia), and it is recognized as the sixth leading cause of death among older people [4]. In 2013, The Royal College of Nursing (RCN) reported that dementia is a challenge for hospitals, due to the fact that around a quarter of hospital beds are occupied by dementia patients. The costs for are considerable for nonresidential care, nursing care, and care home fees and services. If dementia specialist nurses could reduce the hospital stay for older people by even one day, the amount of savings realized could amount to nearly $£ 11,000,000$ nationally [5]. Likewise, the World Alzheimer report shows that "if dementia care was a country, it could be the world's $18^{\text {th }}$ largest economy, ranking between Turkey and Indonesia. Likewise, if dementia care was a company, it would be the world's largest one with annual revenue exceeding Wal-Mart (US\$414 billion) and Exxon Mobil (US\$311 billion). Moreover, costs of informal care- unpaid care e.g., families and others- and the direct costs of social care that is care professionals and in residential home settings- contribute similar proportions (42\%) of total costs worldwide, while direct medical care costs are much lower (16\%).

Correspondence to: Gaire T, Department of Nursing, Innovative College Of Health Science,

Kathmandu, Nepal, Tel: 01-4485376.; E-mail: taragaire33@gmail.com

Received: April 16, 2020, Accepted: June 12, 2020, Published: June 19, 2020

Citation: Pathak KP, Gaire T (2020) Dementia in Nepal: Early Recognition Knowledge, Management Barriers and Practices of Registered Nurses - A Pilot Study. J Gerontol Geriatr Res 9: 512. doi: 10.35248/2167-7182.20.9.512

Copyright: $@ 2020$ Gaire T, et al. This is an open-access article distributed under the terms of the Creative Commons Attribution License, which permits unrestricted use, distribution, and reproduction in any medium, provided the original author and source are credited. 
On the other hand, in low-, middle- and high-income countries, the costs for dementia equate to only $1 \%, 10 \%$ and $89 \%$ respectively. So, it is said that nearly $70 \%$ of the worldwide costs go to North America and western EU. In comparison, this accounts for the much lower costs per person in lower income countries-\$868 in low income countries, $\$ 3,109$ in lower middle income, $\$ 6,827$ in upper middle income and $\$ 32,865$ in high income countries" [6-8].

Registered Nurses and team are crucial part of management and carry the responsibility of providing nursing care to multi morbid and seriously ill residents, as well as developing staff competencies [9]. A nurse works with people in their homes, in care centers and in hospitals to help them manage their health condition, identify the early stages of dementia, and form management strategies for patients and their care givers. Through their wide range of skills and knowledge, RNs increase family and patient awareness of the early identification and the negative impact on people with dementia and their care givers [10].

Registered Nurses need multiple competencies, which demand experience and evidence-based knowledge. To be competent in their field consultative role they need to know how to teach, lead, delegate, and supervise the staff [11]. This wide range of knowledge is essential for dealing with complex situations. Registered Nurses must be able to solve practical problems and to identify the differences between nursing and social care. There are some factors that impede an RN's potential to conduct research or to develop their competence in health care [12].

In 2011, the RCN published five principles for improving dementia care in hospital settings, which were: environment, hospitals, staffs, partnership and individualized care plans. Dementia should be treated as a component of wellbeing in the hospitals to achieve early identification and assessment. Additionally, skilled staff with adequate time for patient care, palliative care, nutrition, individualized care plans, rehabilitation and person-centered care is other significant principles. At the end of their life, two thirds of patients with dementia spend their final years in hospital [13]. Therefore, the ability to provide excellent care to dementia patients in the hospital setting is a significant issue [4].

UK research has shown that dementia patients die while under hospital care due to the slow progression of the disease and the resulting prolonged duration of the care process [14]. Sampson et al. showed that in the UK, hospital patients with dementia receive less palliative care compared to similar individuals without dementia [15]. However, for dementia patients, palliative care is even more necessary. Guidelines have been drafted to assist nursing homes in developing policies and practices for end-of-life care for residents with dementia [16].

Dementia increases the disability rate among the elderly and creates the problems for both caregivers and the person afflicted [17]. Often, in later stages of dementia, it becomes more complicated for the family members and caretakers as they need the additional expert care from the specialized, trained professionals. The care of elderly people with dementia is anticipated to increase in Nepali society due to increased longevity and the disintegration of joint families. There is emerging evidence that the dementia problem is gradually expanding as a future crisis and a national challenge and will soon be marked by a significant growth in its elderly population, similar to Ireland [18,19]. However, according to the Nepal nursing council, as of 12, March 2017, there were 43,149 registered nurses [20]. At present in Nepal, the nursing care at psychiatric/mental based hospitals is a practical solution. Key roles are mental health resources for the detection of cognitive impairment and diagnosis of dementia. Also, there is a smaller number of mental health professionals (psychiatrists $0.13,0.27$ nurses and psychological providers 0.19 in 100,000 populations) that do not meet WHO standardization [18].

That is suggested to have 288, 2,928, and 2,549 respectively. This figure is lower than number of nurses in either the United Kingdom $(400,000)$ or the United States' $(700,000)$ (US. Bureau of labour statistics). However, the global nursing shortage is rising due to an increasing level of job dissatisfaction and burnout [21].

In 2011 , the elderly rate increased rapidly and reached $8.2 \%$ of total population, yet the government has placed no higher priority on identifying the issues associated with managed care for the aging or implementing a senior citizen act for wellbeing [22]. In Nepal, there is virtually no awareness of dementia amongst the public, healthcare professionals, or policy makers. Additionally, neither dementia-related care and management nor trained nurses are available [23]. The illness places a heavy burden on both the elderly patient and their relatives. Scientifically, this is the first step to identify the RNs' actual level of dementia preparedness. It can be estimated that currently about 135,000 people are suffering from some kind of dementia in Nepal [18]. This figure is likely to double every 20-year, which indicates a rate of increase similar to that of western countries. For example, in Greece in 2010, the estimated number of dementia cases was 196,000 to 365000 , in UK 800,000 people, in Belgium the Qualidem study (1999-2006) indicated 170,000 people have suffered 24-25. ButAlzheimer Europe (2019) estimated this number will increase from 213,678 in 2018 to 356,458 in 2050 , The overall numbers of people with dementia will almost double from 1,031,396 in 2018 to 1,977,399 in 2050, in Belgium The overall numbers of people with dementia will almost double from 192,926 in 2018 to 360,766 in 2050[26]. These ratiosshow the dementia is significantly increasing in every nations and it is being being global burden issue [27].

There is a need for educational programs and health-care policies that help to increase awareness of dementia in nursing practice, management and care, thereby improving the care provided to demented people [28]. The main objective of this study is to identify the key concepts in dementia practice, management, and the level of care provided by the Registered Nurses in nursing homes. What is the experience status of Registered Nurses and are they prepared to address the forthcoming dementia issues? As the demand for more nursing homes and additional staff increases, Registered Nurses may lack the practical knowledge and competency required to provide nursing home care [29,30].

\section{RESEARCH METHODOLOGY}

\section{Study site and sample}

To meet the aims of the study we conducted a self-administered questionnaire to explore the registered nurses broad aspects regarding their dementia care management status in Nepalese Nursing homes. This study investigated several crucial thematic issues on dementia patient's management and care knowledge. 
This purposive study was, by the city. For this study, two nursing homes were selected that were established recently with high infrastructures. These two nursing homes were newly established with fifty beds, and were located in the capital city of Kathmandu metropolitan, with high infrastructures, so it was not apparent which patients had a low-income source for health checkups. A for-profit company owns the facilities, which means patients pay for medical health services. There was variety of clinical care settings including Out Patients Department, Intensive Care Unit/ Operation Theater ward, general ward, emergency ward, medical ward, gynecological ward, orthopedic ward, pediatric ward, and psychiatric ward. The intention was to choose these nursing homes and study the practices of their Registered Nurses to determine a number of factors: their level of awareness of dementia; how they cope with dementia issues within their practice, and the difficulties the Registered Nurses encounter in providing dementia care and management services within the nursing homes.

Only female nurses participated in the study because there is currently no practice for male nurses in hospitals. Registered nurses were recruited from varieties of clinical care settings including outpatients departments ward, Intensive care Unit, Operation theatre, general ward, emergency ward, medical ward, gynecological ward, orthopedic ward, pediatric ward, psychiatric ward. A purposive sampling approach identified 44 Registered Nurses from 66 participants (response rate 29.04\%). Participants were asked to complete an English-language questionnaire, which was distributed and collected by the Head Nurse in each unit over a two-month period. Twenty questions were posed to the first nursing home and 24 questions were posed to the second. The Registered Nurses were not required to complete the questionnaire, but it was distributed to those who were interested. There were no requirements for the level of experience, but all RNs were licensed. In the end, 22 of the questionnaires were returned blank. More than half of the RNs declined to complete the questionnaire, citing reasons such as "it is a new issue in the nursing home setting", or "it is not an area of concern in these nursing homes and we are not psychiatric nurses”.

Because of the increased demand for geriatric nursing care and the associated issues, all RNs were employed full-time in different wards, even if they were not planning on a career in geriatric nursing care. As a result, many $\mathrm{RNs}$ declined to complete the questionnaire, which fell short of the goal of interviewing every nurse on every shift. Due to the time limitation, the strict rules and regulation of private nursing homes and the RNs' reluctance to participate, we could not collect sufficient data from these two nursing homes. Also, there were 86 employees (Registered Nurses) in these two nursing homes. A minimum survey was needed to create new public policy and increase public alertness. However, this small scale-research will still help further the understanding of the clinical and managerial aspects of nursing homes Registered Nurses.

\section{Measurement}

We developed and validate the questionnaire with multiple processes before starting study. Firstly, we reviewed the literature identifying the goals and drafted set of questions. Secondly we revised the questionnaire set. Thirdly, confirming face and content validity and revised the questionnaires. Fourth, discussed with the clinical experts from the same hospitals as author given value to the experts on the basis of his/her popularity with in 2 and half months.
The questionnaires prompted respondents to answer a dementia quiz: concerning diagnosis, epidemiology, medication and management of dementia difficulties. There were multiple-choice selections for each question, including a "do not know" option. In part one, 18 questions assessed current practices in dementia care and in the second part, seven questions were related to dementia epidemiology quiz. Two questions related to medication and its uses and effects on patients. One question was related to the multidimensional nature of dementia diagnosis. Another question concerned the prevalence of dementia and last two questions were essay questions that prompted respondents to share their experiences of dementia care, management, and difficulties within their work. The objective was to assess the RN's knowledge, barriers and practices concerning the management and care of dementia patients.

\section{Data analysis}

The questionnaire format was similar to Turner's et al. [31]. These questions were coded according to the Statistical Package for the Social Sciences, SPSS version 20. Our data information used frequencies information with percentages, mean, and standard deviation and we compared to related research and essay responses were summarized manually in descriptive way.

\section{RESULTS}

This is the first study to examine the care and management of dementia knowledge, barriers and practices among $\mathrm{RNs}$ in nursing homes. In this section, data are presented under the following sub titles; Knowledge of Dementia, Dementia Diagnosis Practice, Most diagnosed Issue, Awareness of Dementia Care Services, Obstacles to Management and Care. All participants held nursing license and very few had less knowledge or experience with nursing care management. Table 1 shows the least knowledge of dementia. No significant associations were found between the age of the $\mathrm{RNs}$ and knowledge or experience with dementia. Only a very few were aware of dementia issues, however, they have not yet received any form of dementia training.

\section{Knowledge}

The total mean score of participants' dementia care and management knowledge was very low (mean=3.54, $\mathrm{SD}=1.82$ ). Responses to the knowledge quiz are shown in Table 1 . This table shows that the lowest dementia quiz score was 4 (9.15) in question number 4 and the highest quiz score was $30(68.2 \%)$ in response to question number 2 .

\section{Views regarding on dementia diagnosis}

The result shows that most diagnostic practices are performed by physicians 16 (36.4\%) upon first clinical visit and subsequently confirmed by the neurologist $13(29.5 \%)$. This is compared to dementia diagnosis by GP colleague $6(13.6 \%)$, old age psychiatric 5 (11.4\%), geriatrician $13(29.5 \%)$ are the sub diagnostic role on the basis of registered nurses' response.

\section{Estimation of dementia diagnosis and number of visits in a typical month}

These Registered nurses reported that in their experience, diagnosis 
Table 1: Dementia quiz answers giving by $\mathrm{RNs}(\mathrm{N}=44)$

No.

1

When the Alzheimer disease (AD) recognized that is most common cause of dementia?

A. 1907 s B. $1907 \mathrm{~s}$

Which is not the risk factor of cardio-vascular disease?

2

A. Hypertension B. Atrial fibrillation

C. Ebixa D. Coronary artery disease

Correct percentage

$30(68.2 \%)$

The most common types of dementias of Alzheimer disease takes account for approximately:

$$
\text { A. } 50-70 \% \text { B. } 20-30 \%
$$

C. $30-50 \%$ D. None of them

When a patient develops a sudden onset of confusion, disorientation, and inability to sustain attention, this presentation is most consistent with the diagnosis of:

4

A. Alzheimer's disease B. Acute confusional state

C. Major Depression D. Vascular dementia

Which of the following clinical findings best differentiates vascular dementia from Alzheimer's?

5

A. Word finding problem B. Short term (2-minute span) visual memory loss

C. Stepwise disease course D. Presence of depression.

The effect of anti-dementia drugs is to:

A. Temporarily halt the disease in all cases.

B. Temporarily halt the disease in some cases

Temporarily halt the disease in some cases but often causing liver damage.

Permanently halt the disease in some cases.

Which one is correct?

A. Mild dementia shows-20-24 out of 30 points MMSE score.

Moderate dementia shows-9-20 out of 30 points MMSE score.

Severe dementia shows- 6 out of 30 points MMSE scores.

None of them.

A physical disease which causes brain cells to die, plaques disrupt message within brain is called Alzheimer disease.

A. Word finding problems

8 a.

B. Short term (2-minute span) visual memory loss.

C. Stepwise disease course

D. Presence of depression.

$8 b$. Multiple little strokes have damaged particular areas of the brain is called Vascular disease.

A) Yes. B) No. C) Do not know.

Abnormal lumps called Lew Body Bodies develop inside nerve cells in the Brain. People with LBD often have

$8 c$. delusions, stiffness, tremors, visual hallucinations is called LBD.

A) Yes. B) No. C) Do not know.

A progressive disorder of the central nervous symptoms is characterized by stiffness in joints and limbs,

$8 \mathrm{~d}$. tremors difficulty to movement, speech.

A) Yes. B) No. C) Do not know.

Rivastigmine tartrate (Exelon $\left.{ }^{\circledR}\right)$ Oral; approved for:

A. mild and moderate Alzheimer's Disease only

B. moderate, and severe Alzheimer's Disease

C. for mild cognitive impairment

D. None of them

Side effect of Memantine (Namenda $\left.{ }^{\circledR}\right)$ :

A. Headache, Dizziness, Sedation, Agitation, Constipation

10

B. Nausea, vomiting

C. Nausea, vomiting, and diarrhoea

D. None of them

Correct answers: 1 B; 2 C; 3 A; 4 C; 5 C; 6 B; 7 A; 8-a 1, 8-b 1, 8-c1, 8-d 1; 9 B; 10 A.

With all questions also included a 'don't know' option. 
of the dementia patient may take one to sixteen months. However, thirty-four (77.3\%) RNs selected "do not know" in response to the question of the time period of diagnosis in the clinical process. Additionally, 30 RNs (68.2\%) reported they could not identify the exact number of dementia patients in a typical month, but generally speaking, it is their experience that $1-2$ patients per month are examined and diagnosed with dementia.

\section{What problems are mainly diagnosed in the hospitals?}

In this study, $16(36.4 \%)$ registered nurses reported that at their hospital, doctors evaluate suspected dementia patients with behavioral problems, and declare a diagnosis of Parkinson disease, Alzheimer diseases, or Huntington disease. Our data shows the majority of registered nurses 24 (54.5\%) suggested patients and their families consult first with a psychologist to detect dementia, with the remaining registered nurses suggesting other health professionals, including general practitioners 4 (9.1\%), neurologist 7 (15.9\%), and geriatrician 6 (13.6\%).

\section{Response of registered nurses on care services}

Dementia awareness services and location: With respect to dementia memory clinics, local support services, clinical services, and availability of medicine, the RNs knowledge was very low-one third of RNs were unfamiliar with these sectors entirely. In regard to local support for dementia patients, only 18 nurses (40.9\%) were knowledgeable of such services, yet the same percentage $(40.9 \%)$ confirmed that there was no local support for dementia patients. Only $3(6.8 \%)$ could name memory clinics and location, while 27 RNs (61.4\%) knew that there was day care service available.

Awareness of dementia related medicine: There was another subscale to assess the RNs' awareness regarding with the availability of dementia related medicine. As per our data, the majority of RNs (12 or $27.35 \%$ ) were unfamiliar with available dementia medicine. In response to the question, 'Is there available dementia medicine in the market?' ten respondents $(22.7 \%)$ replied that it is very difficult to find. The remaining nine (20.5\%) responded 'No' (not available), and eight (18.2) responded 'Yes' (available).

\section{Obstacles to the care and management of dementia in the hospital}

This section included essay questions. The RNs indicated that the key obstacles to manage and care for dementia patients were: 1) Age of patients, 2) Misunderstand between old age and dementia 3) Misdiagnosis, 4) Late referral by the physicians, 5) Lack of knowledge about dementia among family members, patients and health professionals; 6) High cost; and 7) No special services in the hospital for elderly people. Working with dementia people is very difficult, particularly in regard to the challenges of communicating, wandering, changing behavior, personal hygiene, safety, and the lack of individual care time [4]. It clearly indicates that dementia is a burdensome disease but is still not a highly prioritized public health issue among health professionals, as opposed to other hospital care diseases. Also, neither were they more alert to dementia issues.

\section{Perception of registered nurses}

Geriatric services are not an attractive profession in the nursing industry so they are not well prepared for the demented patients' care and management. It is led by the views of older people, not just by professionals' interests [32]. Michelle \& Jane show that, GP colleagues, nurses feel insufficiently prepared to provide dementia management service [33]. So, primary health care nurses would benefit from improved training in this area, which could lead to improved access to services for people with dementia and their carers. Registered nurses were not getting any training in this study [34]. In one study by the, $71 \%$ of RNs have insufficient training in dementia management and poor awareness of the support services available for dementia.

It is not only the problem of General practitioners, caregivers and family members, it is problematic for the nurses in respect to the management and care in the hospital settings as well. For that reason, registered nurses suggested taking advantages of the early diagnosis. They were also dissatisfied with the current practice within their hospital. In the present context, there was a higher number of dissatisfied registered nurses (16 or $36.4 \%$ ), compared to the number of satisfied number of registered nurses (5\% or $11.4 \%)$. In contrast, $20(45.5 \%)$ registered nurses had no knowledge of whether the current practice regarding dementia is appropriate or not.

\section{DISCUSSION}

Nursing staff are important in the process of delivering dementia care and interventions because in many cases, nurses are considered the disease experts by patients and caregivers, therefore, the nursing staff's competencies, roles and responsibilities in dementia care is essential. They can play a non-pharmacological and pharmacological role in the management and care of elderly persons [34]. The nurses' continuous visits remain fruitful for institutionalized patients (older patients), pharmacological and non-pharmacological management. The nurses' continuous visits result in better patient care and management, and an improved quality of life of the patients. However, the nursing profession considers it challenging and complicated to assist the patient in the performance of activities that contribute towards improvements to their health [35]. Earlier diagnosis and appropriate detection/ management of dementia is more challenging to GPs and registered nurses due to its hidden controversial symptoms and chronic mental health condition. So, the registered nurses' role in dementia and other associated disorders is a significant institutionalization factor. Institutionalization is not the end-point of better care, but providing excellent care to people with dementia in a hospital setting presents a significant challenge. Our study shows that older patient care was preferred less in this area, than earlier studies conducted in western countries [36]. Our research did not apply any specific scale to classify to knowledge - it used scores similar to other institutional research, specifically: "Yes", "No", "Do not know" and "None of them". We do not have a category for nurses who have good knowledge of dementia management and care issues. It is important to note that, in the translation of these results, misinterpretation may occur. These results have been influenced by the respondents' fear and lack of information about how to answer the questions. The RNs' lack of information about dementia could also be attributed to the decreased number of persons with dementia in their practices. All of these RNs were employed in 
nursing homes, where there is no dementia-specific facility beyond the psychology department. Hence, they may have less contact/ practice/experience with dementia patients when compared to other RNs in developed countries. An investigation of the RNs' dementia care and management knowledge would be extremely important to compare it to the dementia care, management and barriers knowledge among other RNs. As previously mentioned, nurses do not receive sufficient information or any formal dementia training during their hospital work hours, which could negatively impact the care and management of people with dementia. Therefore, there is evidence that the level of diagnosis, management, and nursing care for dementia, is considerably lower, than other types of disease among the Nepalese population, based on the RNs' experiences.

Additionally, in our study, nursing care and management was not satisfactory in the nursing homes with RNs. The present trend is for dementia patients to be taken care of by relatives or their spouse at home [37]. Understanding the social burden of dementia and its costs is a crucial issue for future health care and socioeconomic policymakers, as well as the cause for under-detection and treatment. These issues should also be addressed by creating strategies to increase the awareness and training of health professionals, public media and the community [38]. Above all, our data highlighted that the dementia problem is terribly burdensome, but is not prioritized in the public arena.

In our study, registered nurses indicated that the key obstacles to managing dementia are as follows: 1) misconception with elderly and dementia, 2) misdiagnosis by physicians, 3) late referral by physicians and family members, 4) lack of knowledge of dementia issues in the elderly people and 5) high cost for care to the demented patients. Additionally, the availability of memory clinic services, local supported services, clinical services, and availability of medicine was very low. One-third of registered nurses were unknowledgeable regarding this entire sector. Our study result showed dementia care knowledge among nurses was poor (mean $10.8 / \mathrm{SD}=2.0$ ) in southern Taiwan. This was measured by posing 16 questions to 124 nurses. More than half of the registered nurses showed a poor understanding of dementia with delirium, however, there was a higher level of dementia care knowledge among older nurses. But both age and working experience were negatively associated with a reality-oriented approach toward care [39].

The elderly population is growing and there is likely to be an increase in the numbers of people with dementia in an acute hospital setting. The need exists for critical skilled care training for long-term care staff and increased development of dementia care competencies [40]. The findings of Fessey reported that there are significant gaps in general hospital wards in regards to dementia understanding and implications for adult care [41]. Therefore, caring for demented patients presents a significant challenge in an acute hospital setting. Nolan explored nurses' experiences of caring for older people with dementia, structural inadequacies of the acute hospital as a dementia care environment, and the resulting challenges and complexities of the care experience. Despite this, nurses weighed their ability to meet identified needs against the specific needs of people with dementia and the environmental effects of the acute setting on [42]. That is why, focused on effective performance of a wide range of nursing activities that will be effective in providing knowledge, skills, traits, motives and attitudes with the elderly [43,44].

This study revealed that nurses felt insufficiently prepared to provide appropriate services and would take no action regarding suspected dementia. The management of patients with dementia in hospital settings is a challenge to current health care delivery [45]. The study concluded that nurses would benefit from improved training in this area and that this could lead to improved access to services for people with dementia and their carers.

At last, the findings suggested the need for educational programs/ training and health-care policies that increase awareness of dementia in nursing practice and thereby improve the care provided to people with dementia [46]. This paper outlines the problems and solutions that Nepalese nurses within the community need to adopt in order to deal effectively with its diagnosis, care and management. Currently, they are neither aware, nor do they have the competency. Additionally, they have less concern, less knowledge, no updates to the newly established guidelines, and no especial training. Therefore, there is a need for ongoing education of nurses in dementia care that may minimize the unnecessary barriers/conflicts regarding the management of dementia. We would like to suggest that the nurses should be independently and constantly updated, and hospital administration and nursing college/institutions should provide training and guidelines to create greater competencies surrounding dementia disorder [47-49].

\section{KEY POINTS}

1. Educational support programs are needed to improve registered nurses knowledge and confidence with regard to the care and management.

2. There is an urgent need to develop a more systematic approach from registered nurses and a central register of residential, trained dementia nurses and nursing home facilities.

3. The screening procedure is necessary in the early onset period in the community and should address the issue of negative attitudes to dementia care and management.

4. Nepalese registered nurses need to collaborate more with geriatric specialists and Neurologists, to contribute positively to the psychosocial needs of persons with dementia and their caregivers.

\section{LIMITATIONS}

First-there are limitations with the sample size and data analysis. Second-the information is derived from a metropolitan city. Third-the study site is a small (50-bed) hospital. However, it may provide adequate information to the concerned body about dementia that helps to develop a care and management strategy and collaboration between nurses.

\section{CONCLUSIONS AND RECOMMENDATIONS}

Nepalese registered nurses need to have specific dementia assessment tools/guidelines for better care and management. Registered nurses have a crucial part to play for better diagnosis, monitoring and in providing long term support for individuals with dementia. In this regard, especially in Nepal's registered 
nurses case, they need to work more closely with the caregivers, geriatricians, neurologists, social workers,

NGO/INGOs; allied health teams and psychiatric hospital. Many of these aforementioned issues can be addressed via their professional educational development. More priority needs to be focused towards quality development, research and planning, active care, improving collaboration with professional doctors and medical follow up, understanding and responding to behaviour changes, and care and management with screening instruments. Efforts in Nepal need to be directed towards improving financial support, community support, family support, establishing day care home services, memory clinic services with geriatric services, a separate department of dementia in the hospital and in the community. Improvements in dementia knowledge and self-confidence for management and care should lead to an improvement in service delivery in Nepalese hospital with nurses.

\section{Conflicts of Interest}

None.

\section{Acknowledgement}

None

\section{Funding}

No financial support for the research, authorship and publication.

\section{REFERENCES}

1. https://www. Alzheimer's.org. uk/sites/default/files/2018-05/ Counting_the_cost_report. pdf

2. Rizzi L, Rosset I, Roriz-Cruz M. Global epidemiology of dementia: Alzheimer's and vascular types. Bio Med Res Int. 2014; 2014 (3): 908915.

3. Prince M, Bryce R, Albanese E, Wimo A, Ribeiro W, Ferri CP. The global prevalence of dementia: A systematic review and meta-analysis. Alzheimer's Dement. 2013;9(1):63-75.

4. Alzheimer's Association. Alzheimer's disease facts and figures. Alzheimer's Dement. 2013;9(2):208-245.

5. Royal college of Nursing. Dementia: Scoping the role of the dementia nurse specialist in acute care.2013.

6. https://www. alz.co.uk/research/files/World Alzheimer Report-2010. pdf

7. http://www.alz.co.uk/research/statistics

8. https://www.Alzheimers. org. uk/sites/default/files/migrate/ downloads/alzheimers_society_dementia_2012-_full_report.pdf

9. Andersson N, Cederfjäll C, Jylli L, Nilsson-Kajermo K, Klang B. Professional roles and research utilization in paediatric care: Newly graduated nurses' experiences. Scand J Caring Sci. 2007; 21 (1):91-97.

10. Bryans M, Wilcock J. Issues for nurses in dementia diagnosis and management. Nursing Time. 2001; 97(44):30.

11. Perry M, Carpenter I, Challis D, Hopek K. Understanding the roles of registered general nurses and care assistants in UK nursing homes. Jan. 2003; 42(5):497-505.

12. Karlsson I, Ekman SL, Fagerberg I. A difficult mission to work as a nurse in a residential care home - some registered nurses' experiences of their work situation. Scand J Caring Sci. 2009;23(2):265-273.
13. McCarty M, Addington-Hal J, Altmann D. The experience of dying with dementia: A retrospective study. Int J Geriatr Psychiatry. 1997; 12(3): 404-409.

14. Sampson EL, Blanchard MR, Jones L, Tookman JLA, King M. Dementia in the acute hospital: prospective cohort study of prevalence and mortality. Br J Psychiatry. 2009 195(1): 61-66.

15. Sampson EL, Gould V, Lee D, Blanchard MR. Differences in care received by patients with and without dementia who died during acute hospital admission: A retrospective case note study. Age and Ageing. 2006; 35(2): 187-189.

16. Cahill S, Doran D, Watson M. Guidelines for nursing homes delivering end-of-life care to residents with dementia across the island of Ireland. Quality Age Older Adults. 2012;13 (1):60-71.

17. Huang HL, Shyu YI, Huang HL, Chen ST, Li HJ, Shiu S. Factors associated with dementia care practices among community health nurses: Results of a postal survey. Int J Nurse Studies. 2013; 50(9): 1219-1228.

18. Jha A, Sapkota N. Dementia assessment and management protocol for doctors in Nepal. J Nepal Med Assoc. 2013; 52(189):292-298.

19. https://www. lenus. ie/handle/10147/234551

20. Prince MJ, Wu F, GuoY, Robledo LMG, O’Donnell M, Sullivan R, et al. The burden of disease in older people and implications for health policy and practice. Lancet. 2015; 385(9967):549-562.

21. http://nnc.org.np/

22. Chalise HN, Rai SL. Prevalence and correlates of depression among Nepalese rai older adults. J Gerontol Geriat Res. 2013;2(4):1-5.

23. Pathak K,Montgery A. General practitioners' knowledge, practices, and obstacles in the diagnosis and management of dementia.Aging \& Mental Health.2015;19 (10):912-920.

24. Tsolaki M, Fountoulakis C, Pavlopoulos I, Chantzi E, Kazis A. Prevalence of Alzheimer's disease and other dementing disorders in Pilea, Greece. Am J Alzheimers Dis. 1999; 14(3):138-148.

25. https://www. alz.co.uk/research/World Alzheimer Report-2013. pdf

26. Alzheimer Europe. Dementia in Europe Yearbook 2019. Estimating the prevalence of dementia in Europe.2019; 1-188.

27. Furaker C, Agneta N. Registered nurses' views on nursing competence at residential facilities. Leadership Health Servi. 2013; 26(2):135-147.

28. Gerrish K. Still fumbling along? A comparative study of the newly qualified nurse's perception of the transition from student to qualified nurse. J Adv Nurs. 2000; 32(2): 473-480.

29. Greenwood J. Critique of the graduate nurse: An international perspective. Nurs Educ Today. 2000: 20(1): 17-23.

30. Turner S, Iliffe S, Downs M, Wilcock J, Bryans M, Levin E, et al. General practitioners' knowledge, confidence and attitudes in the diagnosis and management of dementia. Age and Ageing. 2004;33(5):461-467.

31. Reed J, Clarke C, Macfarlane A. Nursing older adults. Open University Press, USA. 2012.

32. Waldemar G, Dubios B, Emre M, Scheltens P, Tariska P, Rosser M. Diagnosis and management of Alzheimer's disease and other disorders associated with dementia. The role of neurologists in Europe. European Federation of Neurological Societies. Euro J Neurol. 2000;7(2):133-144.

33. Azermai M, Kane J, Liperoti R, Tsolaki M, Landi F, Passmore AP, et al. Management of behavioural and psychological symptoms of dementia: Belgium, Greece, Italy, United Kingdom. Euro Geriatr Med. 2012;4(1):50-58.

34. Barker S. Vital notes for nurses: psychology. Wiley-Blackwell. 2007; 216. 
35. Shen J, Xiao LD. Factors affecting nursing students' intention to work with older people in China. Nurse Educ Today. 2012; 32(3): 219-223.

36. ZaritSH, Gaugler JE, Jarrott SE. Useful services for families: Research, endings and directions. Int J Geriatr Psych. 1999; 14(3): 165-181.

37. https://www. alz.co.uk/research/WorldAlzheimerReport-2012. pdf

38. Lin PC, Hsieh MH, Lin LC. Hospital nurse knowledge of and approach to dementia care. J Nurs Res. 2012;20(3):197-207.

39. Williams CL, Hyer K, Kelly A, Leger-Krall S, Tappen RM. Development of nurse competencies to improve dementia care. Geriatr Nurs. 2005; 26(2):98-105.

40. Fessey V. Patients who present with dementia: Exploring the knowledge of hospital nurses. Nurs Older People. 2007;19(10):29-33.

41. Nolan L. Caring for people with dementia in the acute setting: A study of nurses' views. Br J Nurs. 2007;16(7):419-422.

42. Ballantyne A, Cheek J, O'Brien B, Pincombe J. Nursing competencies: Ground work in aged and extended care. Int J Nurs Pract. 1998; 4(3):156-165.

43. Zhang Z, Luk W, Arthur D, Wong T. Nursing competencies: Personal characteristics contributing to effective nursing. J Advance Nurse. 2001;33(4):467-474.

44. Kalaria RN, Maestre GE, Arizaga R, Friedland RP, Galasko D, Hall $\mathrm{K}$, et al. Alzheimer's disease and vascular dementia in developing countries: prevalence, management, and risk factors. Lancet Neurol. 2008;7(9): 812-826.

45. Boustan M, Schubert C, Sennour Y. The challenge of supporting care for dementia in primary care. Clin Interv Aging. 2007; 2(4): 631636. Published online 2007 Dec.

46. http://ghdx.healthdata. org/record/nepal-department-health-servicesannual-report-2010-2011

47. Thompson R, Heath H. Dementia: Commitment to the care of people with dementia in hospital settings. 2013; 1-31.

48. Tilly J, Reed P. Dementia care practice recommendations for assisted living residences and nursing homes-phases 1 and 2. Alzheimer Association. 2009; 1-36.

49. Ferri CP, Prince M, Brayne C, Brodaty H, Fratiglioni L, Ganguli M. Global prevalence of dementia: A Delphi consensus study. Lancet. 366(9503), 2112-2117. 